psychopraxis.neuropraxis 2016 · 19:158-163 DOI 10.1007/s00739-016-0343-9

Online publiziert: 7. September 2016 @ ( Der/die Autor(en) 2016. Dieser Artikel ist eine Open-Access-Publikation.

CrossMark
Harald Stefanits ${ }^{1}$ - Tim J. von Oertzen ${ }^{2}$

' Universitätsklinik für Neurochirurgie, Medizinische Universität Wien, Wien, Österreich

${ }^{2}$ Klinik für Neurologie 1, Neuromed Campus, Kepler Universitätsklinikum, Linz, Österreich

\title{
Epilepsie - eine potenziell chirurgisch behandelbare Erkrankung
}

\section{Über nichtinvasive und invasive Abklärung zum neurochirurgischen Eingriff}

hinsichtlich eines epilepsiechirurgischen Eingriffs überwiesen werden. Gerade bei der Temporallappenepilepsie hat sich gezeigt, dass der neurochirurgische Eingriff dem Add-on eines dritten Antikonvulsivums überlegen ist.

\section{Nichtinvasive Abklärung (Phase 1)}

in bei letzteren eine Eingrenzur der epileptogenen Zone nicht immer sicher vorgenommen werden kann. Ätiologisch können der Erkrankung genetische, metabolische, immunologische, vaskuläre oder strukturelle Ursachen zugrunde liegen.

Eine Sonderform der fokalen Epilepsien stellt die Temporallappenepilepsie (TLE) dar. Sie ist ein durch typische klinische, radiologische und elektrophysiologische Charakteristika gekennzeichnetes Syndrom. An Risikofaktoren für die TLE sind komplizierte Fieberkrämpfe im Kleinkindalter, Schädel-Hirn-Trauma, Meningitis, peripartale Asphyxie oder intrakranielle Tumoren zu nennen.

Etwa die Hälfte aller Patienten mit fokaler Epilepsie entwickeln im Laufe ihrer Erkrankung eine Therapieresistenz, welche per definitionem bereits ab einem Nichtansprechen auf zwei First-line-Antikonvulsiva erreicht ist. Liegt als Pathologie ein Kavernom vor, ist die medikamentöse Therapieresistenz bereits nach einem ausdosierten Antikonvulsivum gegeben. Therapieresistente Patienten sollten nach aktuellen Studienerkenntnissen in ein epilepsiechirurgisches Zentrum zur Evaluierung

Die Grundpfeiler der nichtinvasiven Abklärung im prächirurgischen Setting bilden eine Kombination aus Anamnese, klinischer Untersuchung, Elektroenzephalographie und Bildgebung.

Die detaillierte Anamnese muss auf jeden Fall Angaben zur Anfallshistorie, Anfallstypen und -frequenz, Familienanamnese hinsichtlich Epilepsie, Risikofaktoren sowie auslösende Faktoren wie Flackerlicht oder Schlafentzug enthalten. Die Fremdanamnese mit Angehörigen ist hier essentiell, da bei vielen Epilepsiesyndromen eine Amnesie für die Zeit des Anfalls und die postiktale Phase vorliegt. Außerdem ist ein ausführlicher neurologischer Status $\mathrm{zu}$ erheben.

Das simultane Video-EEG-Monitoring wird über mehrere Tage unter Reduktion der antikonvulsiven Medikation durchgeführt. Außerdem werden unter kontrollierten Bedingungen anfallsprovozierende Flackerlicht- und Hyperventilationsstimulationen vorgenommen.

Das Ziel des Monitorings ist es, zwei bis drei für den Patienten typische fokale Anfälle aufzuzeichnen, die schließ- lich auf klinische und elektrophysiologische Charakteristika hin untersucht werden. So soll einerseits das Epilepsiesyndrom definitiv charakterisiert und andererseits eine Lateralisation und Lokalisation der Anfallsursprungszone ermöglicht werden.

\section{Strukturelle Bildgebung mittels 3T-MRT}

Zur Detektion von anfallsauslösenden Läsionen im Gehirn ist eine Magnetresonanztomographie Mittel der Wahl, die nach aktuellem Stand der Wissenschaft mit einer Feldstärke von 3 Tesla durchzuführen ist. Bei der Sequenzauswahl sollte man sich an die "essential six" halten, welche epileptogene Läsionen mit einer sehr hohen Sensitivität detektieren können. Für die Temporallappenepilepsie ist eine Ausrichtung der koronaren Schnittbilder orthogonal zur anatomischen Längsachse des Hippocampus erforderlich. Neben dem epilepsiespezifischen MRT-Protokoll ist auch die Befundung durch einen in Epilepsie erfahrenen Neuroradiologen von hoher Wertigkeit. Daher sollte diese Bildgebung vornehmlich in Epilepsiezentren durchgeführt oder dort zumindest befundet werden [1-3].

Ein besonderes Augenmerk wird von der Neuroradiologie auf Tumoren, Gefäßfehlbildungen, angeborene Hirnstrukturveränderungen, posttraumatische Narben und das typische Charakteristikum der Temporallappenepi- 


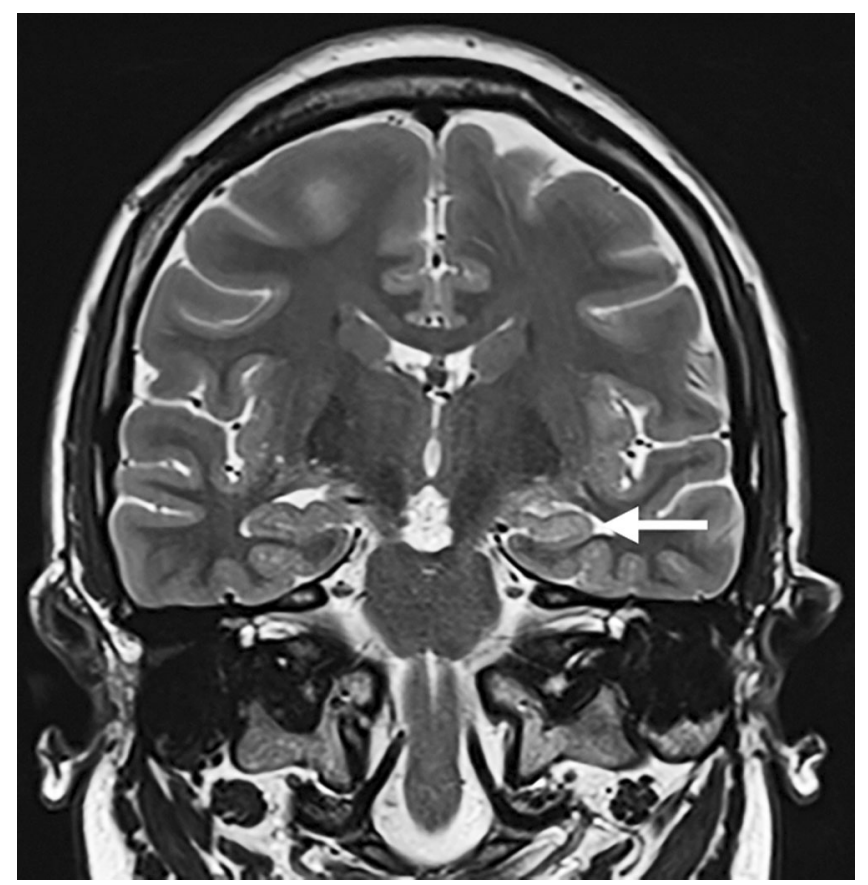

Abb. 1 ॥ 20-jährige Patientin mit seit 3 Jahren bestehender Temporallappenepilepsie, klinisch und im EEG links lateralisiert. Anamnestisch Fieberkrämpfe im Kleinkindalter. Im MRT zeigt sich eine Signalaufhellung im T2-gewichteten koronalen Schnittbild sowie eine Volumenabnahme des Hippocampus als Hinweis auf eine Ammonshornsklerose links. (@ Neurologie 1/Kepler Uniklinikum Linz)

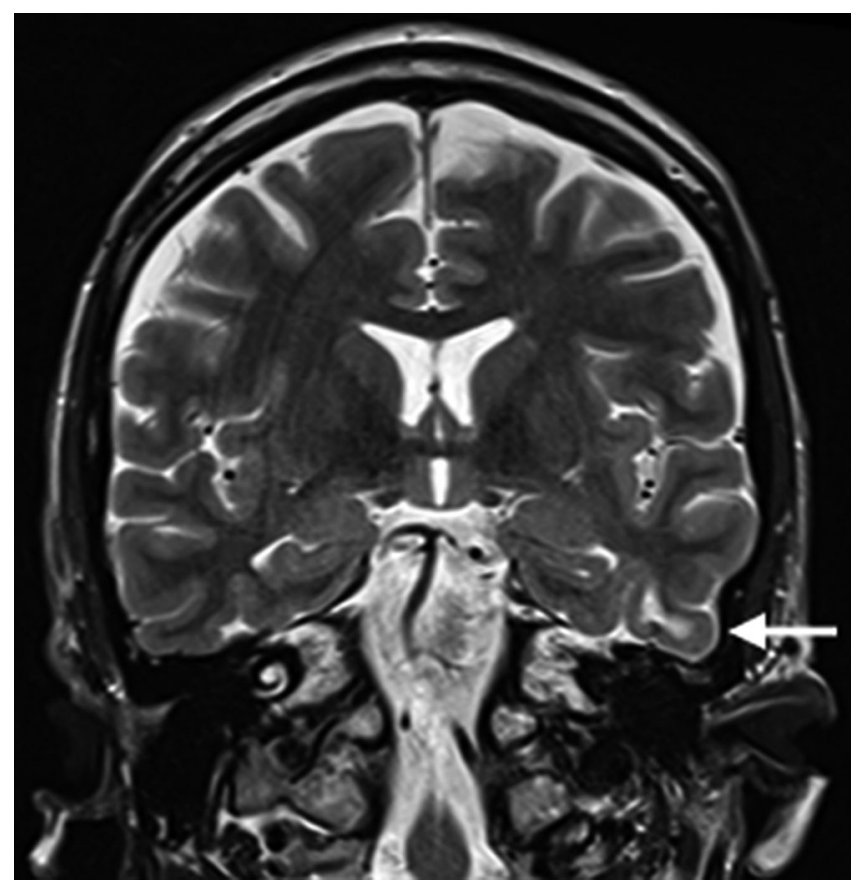

Abb. 2 × 56-jährige Patienten mit seit einigen Monaten bestehender Temporallappenepilepsie links. Im MRT findet sich schließlich im Gyrus temporalis inferior eine subkortikale Signalveränderung, histologisch konnte ein Gangliogliom diagnostiziert werden. (@ Neurologie 1/Kepler Uniklinikum Linz) lepsie, die Ammonshornsklerose, gelegt (- Abb. 1, 2 und 3).

\section{MR-Postprocessing}

Trotz immer besser werdender Bildqualität und Detailtreue kann bei bis $\mathrm{zu}$ 35-40\% der Patienten keine epileptogene Läsion im MRT gefunden werden. Mithilfe spezieller Software kann ein MR-Postprocessing durchgeführt werden, das voll automatisiert Kortexdicke, Grau-Weiß-Differenzierung sowie die inselartige Verteilung von grauer Substanz misst und grafisch sowie numerisch suspekte Regionen definiert. Die anschließende kombinierte Analyse mit dem strukturellen MRT erhöht die Detektionswahrscheinlichkeit von Läsionen deutlich (• Abb. 4).

\section{Funktionelle Bildgebung}

Eine interiktale FDG-PET (Positronenemissionstomographie mit 18F-Fluordesoxyglukose) weist in epileptogenen Arealen einen reduzierten Tracer-Uptake auf und kann somit in der Lateralisation und Lokalisation unterstützen.

Die SPECT (Single-Photon-Emissions-Computertomographie) kann ebenfalls bei der Eingrenzung der epileptogenen Zone unterstützen. Ein radioaktiv markierter Tracer wie HMPAO (99mTcHexamethylpropylenaminoxim), der während eines Anfalls intravenös verabreicht wird, reichert sich in der aktivsten Hirnregion an. Die resultierenden Bilder werden mit interiktalen SPECT-Bildern sowie mit einem strukturellen MRT digital verrechnet (SISCOM) [4].

Zur Lateralisation sowie Lokalisation von eloquenten Regionen wie Sprache, Motorik oder Gedächtnis kann eine funktionelle Magnetresonanztomographie durchgeführt werden.

\section{Neuropsychologie/Psychiatrie}

Eine neuropsychologische Testung erfasst den kognitiven Status der Patienten vor einer Operation, um einen Ausgangswert für die postoperative kognitive Entwicklung zu definieren. Eine psychiatrische Anamnese, insbesondere im Hinblick auf eine vergangene oder gegenwärtige Depression, kann Prädiktoren für das postoperative psychiatrische Ergebnis geben.

\section{Invasive Abklärung (Phase 2)}

Ergibt die gesamte Diagnostik der Phase 1 keine konklusive Anfallsursprungszone, die einem epilepsiechirurgischen Eingriff zugänglich ist, kann in ausgewählten Fällen zur Lateralisation oder zur Eingrenzung der Anfallsursprungszone eine invasive Abklärung angeschlossen werden. Dabei werden vom Neurochirurgen der Kortexoberfläche aufliegende subdurale Streifen- oder Plattenelektroden oder ins Hirnparenchym eingebrachte Tiefenelektroden temporär implantiert. Über diese wird - kabelgebunden - das EEG abgeleitet, welches direktere Informationen als das Oberflächen-EEG bietet, da Schädelkalotte und Galea als „Störfaktoren“ wegfallen. Bereits bei der Planung der Elektrodenlage muss penibel auf anatomische und funktionelle Gegebenheiten des individuellen Gehirns geachtet werden sowie eine Hypothese formuliert 
werden, die den Anfallsursprung bereits vor Implantation auf ein abgrenzbares Areal einschränkt.

Subdurale Streifen- und Plattenelektroden haben den Vorteil, dass sie große Kortexareale abdecken und durch die Elektrodenverteilung eine Ausbreitung in Ort und Zeit an der Hirnoberfläche aufzeichnen können. Subduralelektroden werden über Bohrlochtrepanationen oder - je nach Elektrodenanzahl Kraniotomien auf die Hirnoberfläche aufgebracht.

Tiefenelektroden können eingesetzt werden, um tiefer gelegene Hirnbezirke wie etwa den medialen Temporallappen oder die Insel zu erreichen. Sie werden stereotaktisch neuronavigationsgestützt in zuvor definierte Hirnareale implantiert.

Postoperativ setzt sich die Phase 2 analog der Phase 1 mit Video-EEG-Monitoring fort. Die Elektroden können üblicherweise nach einigen Tagen - sobald ausreichend Anfälle abgeleitet wurden entfernt werden. Durch kortikale Elektrostimulation kann zudem ein Mapping funktioneller Areale durchgeführt oder ein Auslösen von Nachentladungen oder Anfällen hervorgerufen werden.

\section{Epilepsiechirurgie}

Je nach Art des Epilepsiesyndroms sowie den Ergebnissen der prächirurgischen Evaluierung kann ein kurativer oder palliativer epilepsiechirurgischer Eingriff indiziert werden. Diese Operationstechniken sind epilepsiechirurgischen Zentren vorbehalten, da sowohl die Planung als auch die Durchführung der Operation viel Erfahrung und ausgereifte technische Fertigkeiten sowie ein eingespieltes Team und eine entsprechende technische Ausstattung im Operationssaal benötigen.

\section{Standardtechniken}

Das strukturelle Charakteristikum der Temporallappenepilepsie ist die mesiale temporale Sklerose, auch bekannt als Ammonshorn- oder Hippocampussklerose (AHS). Diese ist häufig sowohl im MRT als auch in der histologischen Untersuchung zu erkennen und durch einen

psychopraxis.neuropraxis 2016 · 19:158-163 DOI 10.1007/s00739-016-0343-9

(c) Der/die Autor(en) 2016. Dieser Artikel ist eine Open-Access-Publikation.

\section{H. Stefanits · T. J. von Oertzen \\ Epilepsie - eine potenziell chirurgisch behandelbare Erkrankung. Über nichtinvasive und invasive Abklärung zum neurochirurgischen Eingriff}

\section{Zusammenfassung}

Die Epilepsie ist eine der häufigsten neurologischen Erkrankungen im Erwachsenenalter. Behandlung der ersten Wahl für fokale Epilepsien ist die medikamentöse Therapie. Allerdings sollte bereits nach einem nicht Ansprechen auf zwei First-line-Antikonvulsiva die Zuweisung in ein epilepsiechirurgisches Zentrum zur Evaluierung eines möglichen neurochirurgischen Eingriffs erfolgen. Die nichtinvasive prächirurgische Phase-1Abklärung beinhaltet Anamnese inklusive Fremdanamnese, klinische Untersuchung, strukturelle und funktionelle Bildgebung, simultanes Video-EEG-Monitoring sowie eine neuropsychologische Untersuchung. Kann die epileptogene Zone nicht ausreichend eingegrenzt werden, kann eine invasive
Phase 2 mit Implantation von subduralen Streifen- oder Plattenelektroden sowie stereotaktisch implantierten Tiefenelektroden angeschlossen werden. Wird die Indikation zum epilepsiechirurgischen Eingriff gestellt, ist eine penible Planung der Technik sowie des Resektionsausmaßes essenziell, um das bestmögliche Ergebnis für den Patienten zu erzielen. Bei Patienten mit Temporallappenepilepsie kann durch eine Operation in 60-80\% der Fälle Anfallsfreiheit erzielt werden.

Schlüsselwörter

Epilepsie · Neurochirurgie · Ammonshornsklerose - Langzeitepilepsieassozierte Tumoren (LEAT) · Fokale kortikale Dysplasie (FCD)

\section{Epilepsy - a disorder that is potentially treatable with surgery. Noninvasive and invasive evaluation for neurosurgery}

\section{Abstract}

Epilepsy is one of the most frequent neurological diseases in adults. First line therapy consists of antiepileptic drugs. However, up to $50 \%$ of patients with focal epilepsy do not adequately respond to medical therapy. After failure of two first-line drugs, patients should be referred to a comprehensive epilepsy center for evaluation of the possibility for epilepsy surgery. The noninvasive phase 1 includes history with eye witness report, clinical examination, structural and functional imaging techniques, simultaneous video-EEG monitoring as well as a neuropsychological examination. An invasive phase 2 can be added in cases with incongruent results. Subdural strip and grid electrodes as well as stereotactically implanted depth electrodes give more detailed information on seizure origin and spread. Detailed planning of the surgical approach is of utmost importance. In patients with temporal lobe epilepsy, seizure freedom can be achieved in $60-80 \%$ of cases after epilepsy surgery.

\section{Keywords}

Epilepsy · Neurosurgery · Hippocampal sclerosis - Long-term epilepsy-associated tumor (LEAT) · Focal cortical dysplasia (FCD)
Verlust von Nervenzellen und eine reaktive Astrogliose gekennzeichnet. Auch für die AHS werden verschiedene Subtypen (HS Typ 1-3 und no-HS) unterschieden, die eine unterschiedliche prognostische Aussagekraft im Hinblick auf die zu erwartende Anfallsfreiheit nach einer Operation haben. - Allerdings ist diese Aussage erst durch die Histologie nach der Operation möglich. Die beste Prognose weist schließlich die klassische Ammonshornsklerose (ILAE Typ 1) mit einem Nervenzellverlust in allen CA-
Sektoren mit einer relativen Aussparung von CA2 auf, die schlechteste die nicht läsionelle Temporallappenepilepsie (ILAE no-HS) [5].

Die chirurgische Therapie der Temporallappenepilepsie mit und ohne mesiale temporale Sklerose konzentriert sich auf zwei Standardresektionstechniken, die je nach Ausdehnung der epileptogenen Zone zur Anwendung kommen. Die selektive Amygdalohippokampektomie wird über eine nach temporal fokussierte pterionale Kraniotomie und schließlich 


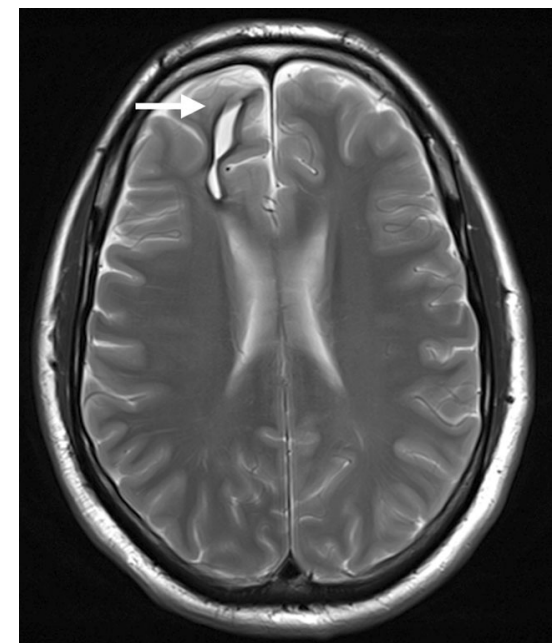

Abb. 3 A 34-jähriger Patient mit Hämophilie. Frontallappenepilepsie mit häufig sekundär generalisierenden Anfällen seit dem Kindesalter. Das MRT zeigt eine posttraumatische Narbe von der Spitze des rechten Seitenventrikels bis nach frontopolar reichend. (๔) Neurologie 1/Kepler Uniklinikum Linz)

einen transsylvischen Zugang, also eine Spaltung der Sylvischen Furche, durchgeführt. Dabei werden Amygdala und Uncus und die gut erreichbaren, meist rostralen zwei Drittel des Hippocampus, entfernt. Diese Methode kommt häufig bei im MRT identifizierter Ammonshornsklerose mit einer elektrophysiologisch auf den mesialen Temporallappen eingrenzbaren Anfallsursprungszone zur Anwendung. Wenn diese über den Temporalpol auf den lateralen Temporallappen ausgedehnt ist, kann eine anteromediale Temporallappenresektion (AMTR) durchgeführt werden. Dabei werden über eine frontotemporale Kraniotomie und eine Kortikotomie der Temporalwindungen sowohl die medialen Anteile als auch der Pol und die rostral gelegenen Anteile des lateralen Temporallappens entfernt. Hier ist vor allem auf der sprachdominanten Hemisphäre speziell auf die Wernicke-Region und den Fasciculus arcuatus zu achten, da Verletzungen dieser Strukturen zu sensorischen oder Leitungsaphasien führen.

\section{Individuell angepasste Resek- tionen und Diskonnektionen}

Ergibt die prächirurgische Evaluierung eine fokale Epilepsie mit einer definier-
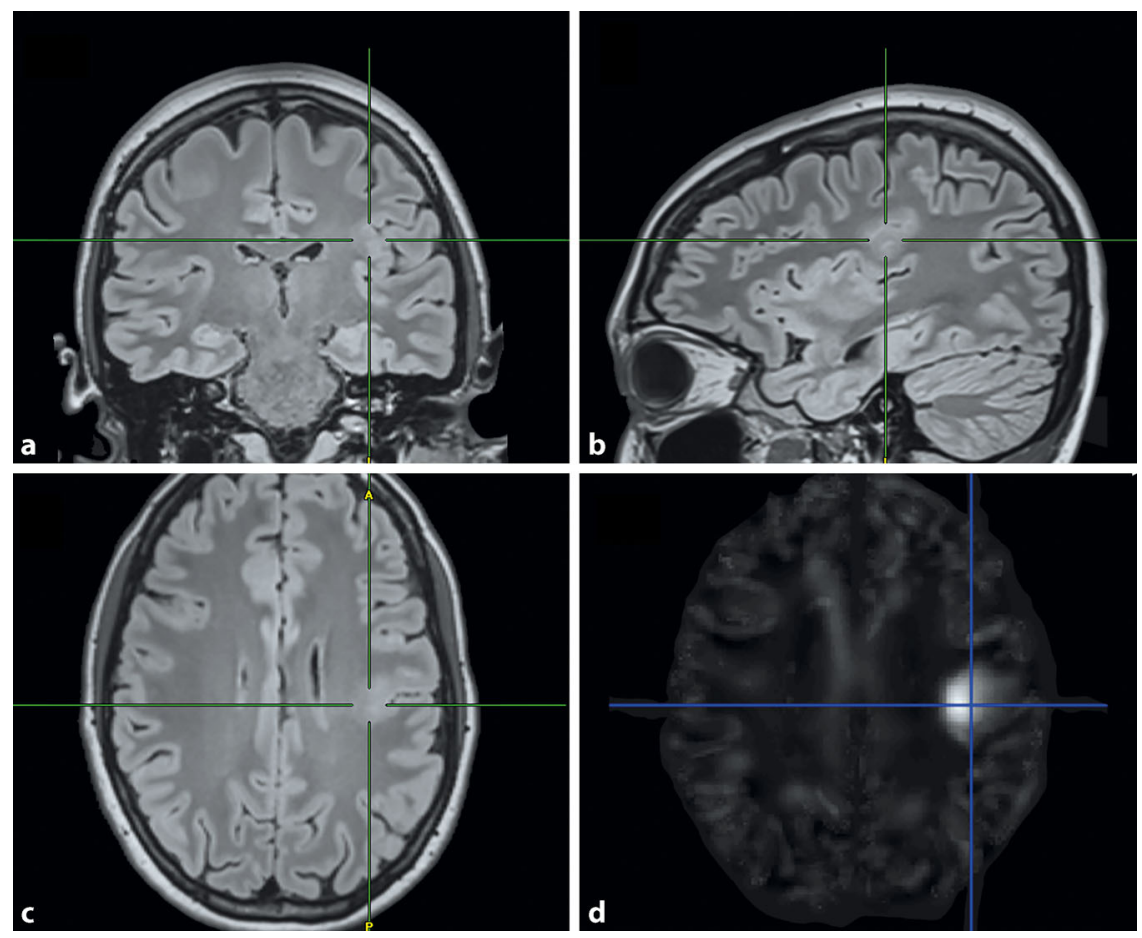

Abb. $4 \Delta$ a-c Bei extratemporalen Epilepsien lässt sich auch im MRT nach Epilepsieprotokoll (FLAIR in 3 Ebenen) oft keine strukturelle Veränderung erkennen. $\mathbf{d}$ Semiautomatisierte Postprocessing-Werkzeuge können „versteckte“ Läsionen mit hoher Sensitivität detektieren. Die Ergebnisse fließen in die weitere Entscheidungsfindung ein. (@ Neurologie 1/Kepler Uniklinikum Linz)

ten epileptogenen Zone, ist in vielen Fällen ein resektiver Eingriff möglich. Handelt es sich bei der zugrundeliegenden Pathologie um einen Tumor, eine Gefäßfehlbildung oder eine umschriebene Störung der Kortexarchitektur, kann oft durch eine Läsionektomie Anfallsfreiheit erzielt werden.

Eine Sonderform der epilepsieassoziierten Tumoren stellen die sog. „long-term epilepsy associated tumors“ (LEATs) dar. Dabei handelt es sich um vorwiegend sehr langsam wachsende, hoch differenzierte neurogliale oder gliale Tumoren, die meist im Temporallappen wachsen und sehr häufig mit einem Anfallsleiden assoziiert sind. Beispiele hierfür sind das Gangliogliom oder der dysembryoblastische neuroepitheliale Tumor (DNET). Bei diesen hirneigenen Tumoren wird die Operation in einer überwiegenden Mehrheit der Fälle nicht aus onkologischen, sondern aus epilepsiechirurgischen Gesichtspunkten geplant und aufgrund des vorwiegend benignen Wachstumsverhaltens das Erreichen von Anfallsfreiheit als Ziel definiert. Die grundlegende Änderung der
Betrachtungsweise vor allem niedriggradiger Gliome in der Neuauflage der WHO-Klassifikation der Hirntumoren aus 2016 wird die Sicht auf die LEATs in den kommenden Jahren sicherlich verändern. Rein histologische Charakteristika rücken in den Hintergrund, während molekulare Marker zunehmend an Bedeutung gewinnen [6].

$\mathrm{Zu}$ den häufig mit Anfällen assoziierten Gefäßfehlbildungen zählen unter anderem die Kavernome und die arteriovenösen Malformationen (AVM).

Die umschriebenen Architekturstörungen des Kortex werden als fokale kortikale Dysplasien (FCD) bezeichnet. Sie sind meist extratemporal, insbesondere frontal anzutreffen und werden von der ILAE in drei Kategorien eingeteilt, nämlich die reinen kortikalen Laminierungsstörungen (Typ 1), FCDs mit dysmorphen Nervenzellen (Typ 2a) und Ballonzellen (Typ 2b) sowie FCDs in Kombination mit assoziierten Läsionen (Typ 3) [7].

In speziellen Fällen muss das Resektionsausmaß schließlich der zugrundeliegenden Pathologie angepasst werden. 
Tab. 1 Ergebnis-Klassifikation der ILAE-beurteilt wird jeweils die Anfallssituation in den $12 \mathrm{Mo}-$ naten vor dem Kontrolltermin (außer 1a)

\begin{tabular}{|ll}
\hline Klasse & Definition \\
\hline $1 \mathrm{a}$ & Anfallsfrei seit der Operation, keine Auren \\
\hline 1 & Anfallsfrei, keine Auren \\
\hline 2 & Nur Auren, keine anderen Anfälle \\
\hline 3 & $1-3$ Anfallstage pro Jahr, mit oder ohne Auren \\
\hline 4 & 4 Anfallstage pro Jahr; bis zu $50 \%$ Anfallsreduktion, mit oder ohne Auren \\
\hline 5 & $\begin{array}{l}<50 \% \text { Anfallsreduktion; bis zu } 100 \% \text { Steigerung der Anfallsfrequenz, mit oder ohne } \\
\text { Auren }\end{array}$ \\
\hline 6 & $>100 \%$ Steigerung der Anfallsfrequenz, mit oder ohne Auren \\
\hline
\end{tabular}

Erstreckt sich das zu resezierende Areal auf der sprachdominanten Hemisphäre bis knapp an die Sprachregion oder ist ein Tumor oder eine FCD nahe einem eloquenten Areal, etwa dem Motorkortex der Präzentralregion, gelegen, kann auch eine Wachoperation durchgeführt werden. Dabei wird der Zugang zum Operationsgebiet in Vollnarkose durchgeführt, aus welcher der Patient schließlich während der Operation gezielt erwacht. Mit einer elektrischen Stimulationssonde können definierte Hirnregionen von etwa $0,5-1 \mathrm{~cm}^{2}$ Größe für die Zeitdauer der Stimulation (wenige Sekunden) ausgeschaltet werden und die Funktion bzw. der Funktionsverlust direkt am wachen Patienten überprüft werden, bevor das Hirnareal entfernt wird. Nach der Stimulation werden die Patien- ten wieder in Vollnarkose versetzt und die Operation zu Ende gebracht. Damit kann sichergestellt werden, dass nur Areale reseziert werden, die keine unmittelbare Funktion innehaben. Eine gezielte Regionalanästhesie im Bereich des Hautschnitts sorgt für eine Schmerzfreiheit in der Wachphase.

\section{Hemisphärotomie}

Vor allem bei Epilepsiesyndromen des Kindesalters wie etwa der RasmussenEncephalitis, dem Sturge-Weber-Syndrom oder nach perinatalen Insulten kann eine Diskonnektion der betroffenen Hemisphäre bei bis zu $80 \%$ der Patienten zu Anfallsfreiheit führen [8].

\section{Palliative Verfahren}

Neben der Kallosotomie, die eine Durchtrennung der die Hemisphären verbindenden Fasern beinhaltet und bei Kindern mit Sturzanfällen Anwendung findet, können bei Patienten mit multifokalen Epilepsien, die nicht einem resektiven Eingriff zugänglich sind, Techniken zur Neurostimulation zur Anwendung kommen. Die etablierteste Methode ist die Vagusnervstimulation. Dabei wird in einer Operation eine Elektrode um den Nervus vagus im Halsbereich gewickelt und mit einem in die Brust implantierten Impulsgenerator verbunden. Über eine pulsatile elektrische Stimulation kann die Anfallsfrequenz bei $30-70 \%$ der Patienten um bis zu $50 \%$ reduziert werden.

Bei der Oberflächen- oder Tiefenhirnstimulation werden Stimulationselektroden nach subdural oder als Tiefenelektroden in Unterkerne des Thalamus eingebracht. Über elektrische Stimulation kann eine Anfallsreduktion erreicht werden, wobei sog. responsive Systeme einer Dauerstimulation überlegen zu sein scheinen.

\section{Postoperatives Follow-up und klinisches Ergebnis}

Je nach zugrundeliegender Pathologie erfolgt ein mehr oder weniger engmaschi-

Hier steht eine Anzeige.

是Springer 
ges Follow-up der Patienten. Bei niedriggradigen Tumoren ist in den ersten Jahren ein dreimonatiges Kontrollintervall mittels MRT einzuhalten, um ein Tumorrezidiv früh erkennen und behandeln zu können. Die epileptologischen Kontrollen erfolgen zumindest im Jahresrhythmus, wobei bei jeder Kontrolle die Anfallsfreiheit beurteilt und anhand der modifizierten Klassifikation nach Wieser (ILAE-Klassifikation) eingeteilt wird (• Tab. 1; [9]). Nach einer entsprechend langen anfallsfreien Periode kann in Absprache mit dem betreuenden Facharzt für Neurologie auch über eine Reduktion oder ein langsames Ausschleichen der antiepileptischen Medikation nachgedacht werden.

Strukturelle Epilepsien aufgrund von LEATs, vaskulären Malformationen oder fokalen kortikalen Dysplasien sind im Falle einer Totalresektion vorwiegend kurabel. Die Erfolgsaussichten für Anfallsfreiheit bei Patienten mit Temporallappenepilepsie liegen bei 60-80\%.

\section{Fazit für die Praxis}

- Fokale Epilepsien sind - auch wenn medikamentös therapierefraktär - potenziell kurativ behandelbare Erkrankungen.

- Bei Versagen von bereits zwei antikonvulsiven Medikamenten, sollte die Zuweisung in ein epilepsiechirurgisches Zentrum zum stationären Video-EEG-Monitoring erfolgen.

- Die therapierefraktäre Temporallappenepilepsie mit struktureller Veränderung im MRT weist eine postoperative Anfallsfreiheit von bis zu $80 \%$ auf.

\section{Korrespondenzadresse

H. Stefanits
Universitätsklinik für
Neurochirurgie, Medizinische
Universität Wien
Währinger Gürtel 18-20,
1090 Wien, Österreich
harald.stefanits@
meduniwien.ac.at

Open access funding provided by Medical University of Vienna.

\section{Einhaltung ethischer Richtlinien}

Interessenkonflikt. H. Stefanits gibt an, dass kein Interessenkonflikt besteht. T.J. von Oertzen erhielt Honorare von Eisia GmbH, Wien, UCB Pharma GmbH, Wien, Novartis Pharma GmbH, Wien, Genzyme Austria $\mathrm{GmbH}$, Wien, Reiseunterstützung von Biogen Idec Austria sowie finanzielle Unterstützung für wissenschaftliche Projekte von UCB Pharma GmbH, Wien.

Dieser Beitrag beinhaltet keine von den Autoren durchgeführten Studien an Menschen oder Tieren.

Open Access. Dieser Artikel wird unter der Creative Commons Namensnennung 4.0 International Lizenz (http://creativecommons.org/licenses/by/4.0/deed. de) veröffentlicht, welche die Nutzung, Vervielfältigung, Bearbeitung, Verbreitung und Wiedergabe in jeglichem Medium und Format erlaubt, sofern Sie den/die ursprünglichen Autor(en) und die Quelle ordnungsgemäßnennen, einen Linkzur Creative Commons Lizenz beifügen und angeben, ob Änderungen vorgenommen wurden.

\section{Literatur}

1. Urbach $\mathrm{H}$, Hattingen J, von Oertzen J, Luyken C, Clusmann H, Kral T, Kurthen M, Schramm J, Blümcke I, Schild HH (2004) MR imaging in the presurgical workup of patients with drug-resistant epilepsy. Am J Neuroradiol 25:919-926

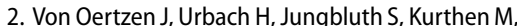
Reuber M, Fernández G, Elger CE (2002) Standard magnetic resonance imaging is inadequate for patients with refractory focal epilepsy. J Neuro Neurosurg Psychiatry 73:643-647

3. Wellmer J, Quesada CM, Rothe L, Elger CE, Bien CG, Urbach H (2013) Proposal for a magnetic resonance imaging protocol for the detection of epileptogenic lesions at early outpatient stages. Epilepsia 54:1977-1987

4. von Oertzen TJ et al (2011) Prospective use of subtraction ictal SPECT coregistered to MRI (SISCOM) in presurgical evaluation of epilepsy. Epilepsia 52(12):2239-2248

5. Blumcke I et al (2013) International consensus classification of hippocampal sclerosis in temporal lobe epilepsy: a task force report from the ILAE Commission on Diagnostic Methods. Epilepsia 54(7):1315-1329

6. Blumcke I et al (2014) A neuropathology-based approach to epilepsy surgery in brain tumors and proposal for a new terminology use for long-term epilepsy-associated brain tumors. Acta Neuropathol 128(1):39-54

7. Mühlebner Aetal (2016)Childhood onsettemporal lobe epilepsy: beyond hippocampal sclerosis. Eur J Paediatr Neurol 20(2):228-235

8. Dorfer C et al (2015) Epilepsy surgery: recent advances in brain mapping, neuroimaging and surgical procedures.J Neurosurg Sci 59(2):141-155

9. Wieser HG et al (2001) ILAE Commission report. Proposal for a new classification of outcome with respect to epileptic seizures following epilepsy surgery. Epilepsia 42(2):282-286
Veranstaltungshinweis

\section{Fortbildungsveranstaltung zum Thema \\ „Der Parkinson-Patient in der Allgemeinpraxis"}

Wann: Mittwoch, 16. November 2016, 19:30 Uhr

Wo: Hotel Courtyard by Marriott Messe Wien, Trabrennstraße 4, 1020 Wien

Vortragender: Prim. Dr. Dieter Volc, Wien

Anmeldung bis 25.10.2016:

MEDahead Gesellschaft für medizinische Information m.b.H.

Tel.: 01/607 02 33-709,

Fax: 01/607 02 33-973,

E-Mail: b.bittner@medahead.at

Im Anschluss lädt die Firma Sandoz GmbH zu einem Buffet ein. 